\title{
Development status of micro seismic monitoring technology in China
}

\author{
Haifeng Chen ${ }^{1}$, Zhendong Lei ${ }^{1}$, Ang $\mathrm{Li}^{2}$, Xixin Wang ${ }^{1}$, Haiqiang Hou ${ }^{1}$ \\ 1 Northeast Petroleum University unconventional oil-gas accumulation-development provincial jointly key \\ laboratory, Daqing, Heilongjiang, China, 163318 \\ 2 Research Institute of exploration and development of Daqing Oilfield, Daqing, Heilongjiang, China, 163712
}

\begin{abstract}
In recent years, the micro seismic monitoring technology have the widespread application in Chinese reservoir geological activity monitoring and mine safety monitoring. The effect of micro seismic monitoring technology is very good. However, in the study of micro earthquake, Chinese research started late relatively with foreign. This paper summarizes the current Development status and the application situation of the Chinese micro seismic monitoring technology.
\end{abstract}

Keywords: - Monitoring of reservoir geological activity Mine safety monitoring Micro seismic

\section{INTRODUCTION}

The principle of micro seismic monitoring technology originated in the monitoring technology of natural earthquake. When the underground strata is rifting and dislocation, fracture will release energy that spread outward in the form of seismic waves. By receiving and monitoring for micro wave can be calculated in real-time location of underground rock fracture ${ }^{[1]}$. Therefore, micro seismic monitoring technology can provide the activity status information of underground rock to geophysical engineers. Micro seismic monitoring technology can be applied to the precursor-monitoring of the volcano and earthquake disaster, as well as rockburst monitoring in the process of exploitation of mineral resources ${ }^{[2]}$. In reservoir development, the micro seismic source determination can judge the Waterflood Front position, In order to provide reliable information for judgment of remaining oil distribution ${ }^{[3]}$. Micro seismic monitoring technology can also determine the location of the crack in the process of hydraulic fracturing ${ }^{[4]}$. Compared with other fracture prediction technique, Micro seismic monitoring technology has higher accuracy ${ }^{[5]}$. In the 80 's of last century, with the development of electronic technology and computer technology, USA began to micro seismic monitoring technology is applied to the development of oil and gas reservoirs, and get good results ${ }^{[6]}$. However, in 2000, China put this new geophysical technology into the domestic reservoir development. Because the micro seismic monitoring technology is very good in accuracy location of strata movement, this technology obtained the rapid development in Application of Chinese geological engineerin ${ }^{\text {g[7] }}$. Along with the increase of mining area of China unconventional hydrocarbon reservoirs, Micro seismic monitoring technology will be a more and more important role in the China unconventional hydrocarbon reservoirs ${ }^{[8]}$.

\section{APPLICATION IN HYDROCARBON EXPLOITATION}

A For the higher accuracy of prediction of the crack and the distribution, Micro seismic monitoring technology is widely applied in many chinese oilfields, such as Daqing oilfield, Changqing Oilfield, Jilin oilfield and the Huabei oil field etc. with the development of normal reservoir in Chinese east gradually mature, unconventional reservoirs shouldering the important mission of sustainable development strategy China energy. The unconventional reservoirs have such features as compactness, low porosity and low permeability, and 
hydrocarbon must move through the crack To form industrial oil flow. But the deal between production well and injection of fracture、stress is the key for efficient development of low permeability fractured hydrocarbon reservoir ${ }^{[9]}$. Micro seismic monitoring technology is a way recognized as the most accurate imaging effect of Fracture prediction ${ }^{[10]}$. Hence, Micro seismic monitoring technology more and more attention Chinese oil field.

B With the eastern China oilfield development level gradually deepened, secondary oil recovery, Tertiary oil recovery has become the main production mode. Look for residual oil will be the main way of these old oilfields to maintain petroleum production. Micro seismic monitoring technology can well predict Waterflood Front, so it is concluded that the remaining oil distribution.

C Therefore, in Chinese, micro seismic monitoring technology in unconventional reservoirs or old oil fields will have a good application prospect. But, at present, Chinese micro seismic monitoring service are provided by foreign oil companies. Study on microseismic monitoring technology in Chinese native has been in the stage of theory ${ }^{[11]}$. Due to more and more technical requirements about micro seismic monitoring technology of China, China will spend more energy in the study of micro seismic monitoring technology. It is absolutely significant to the development of all Chinese oil fields that China should have its own micro seismic monitoring technology.

\section{STUDY OF MICROSEISMIC MONITORING TECHNOLOGY IN CHINA}

In Chinese, study on microseismic monitoring technology is late. In recent years, this technology has been applied to many fields, such as Safety monitoring of mining area, The imaging of layer water injection, Prediction of fracture, Analysis of the oil-water movement. In 2001, Xinglong coal mine according to the distribution of micro seismic events, to explain the distribution of stress field of the underground ${ }^{[12]}$. This is a earlier case of our microseismic monitoring technology in china, but it is complete with Australia cooperation. In 2005, Professor Jiang Fuxing of Beijing Science and Technology University lead the research group of micro seismic monitoring technique to study rock burst in coal mine ${ }^{[13]}$. The study results make application effect of the micro seismic monitoring technology used in chinese mine safety became more mature. The micro seismic monitoring equipment invented with the help and development of the group's research, owned independent intellectual property rights, Monitor different boundary conditions of the overlying strata movement rule. To solve the impact of coal mining liberation layer parameters and high stress region of dynamic positioning prediction problems; In 2006, Jilin University professor Liang Sheng, through the micro seismic monitoring technique, led his team to determine water drive front position of water injection wells in the Qin-Jiatun area, and then study the movement disciplinarian of oil and water and The distribution of remaining oil ${ }^{[14]}$. This study shows that the new geophysical technology in Chinese oil field development will have a good application prospect. In 2006, Wang Zhizhong et al, In Zhuang-19-well area of Changqing Oilfield, Analyse crack distribution characteristics of fractured well Through the research on microseismic fracture monitoring results, This method has important significance for improving the crack test level and the effect of oilfield development ${ }^{[5]}$. The field experiment was carried out in the domestic Daqing, Jilin, North China, Changqing and Zhongyuan Oilfield, satisfactory results have been achieved ${ }^{[2]}$. But the instrument for micro seismic monitoring mostly foreign instruments, And China has been in the micro seismic theoretical research stage. In Stress theory in micro seismic strata, The micro seismic signal denoising and Positioning method of micro seismic inversion and Optimal velocity model, China have invested a lot of energy, And have made good achievements in scientific research ${ }^{[15][16][17]}$.

\section{BASIC RESEARCH}

A Geophone received seismic wave signal of micro seismic, and then through the calculation of computer inversion can determine the position of seismic source. Combining with source 3D statistical model to 
explain the situation of underground fracture, micro seismic monitoring technology can clearly show the distribution and range of the fracture, and guide the oilfield subsequent production development.

B In china, at present, the main methods of micro seismic monitoring are ground micro seismic monitoring method, micro seismic wellhead monitoring method and micro seismic borehole monitoring method. They collected characteristic way makes them have their own advantages, also have their shortcomings. On the ground of micro seismic detection mode, when fracturing layer is deep, placed in the ground detector may not be able to detect micro earthquake, so only is it suitable for that shallow oil and gas reservoirs with low permeability.

C In the micro seismic wellhead monitoring mode, this way can overcomes the difficulty of micro seismic monitoring of deep. However, in the positioning of micro earthquake hypocenter location, only the horizontal coordinate of the source is more sensitive and the focal depth is not sensitive. When the tectonic and sedimentary characteristics of target layer is more complex, the estimation of objective layer depth increased difficulties. So this method is only suitable for low permeability oil gas block that geological tectonic feature layer is simple.

D Method of microseismic monitoring in borehole, as long as the detector is disposed in the monitoring wells near fracturing layer, can be very good to get the horizontal coordinate and depth of information of microseismic source. But this method needs the detector placed in monitoring wells, the cost of this method is very high. in the development of low permeability oil fields, this method is suitable for microseismic monitoring of deep and complicated tectonic geology ${ }^{[18]}$.

\section{APPLICATION PROSPECT}

According to the research results of foreign scholars, Micro earthquakes can be used as passive seismic identification of oil and gas reservoir, and produce different response characteristics according to the presence or absence of the oil and gas layer ${ }^{[19]}$. This kind of research in the China oil field is still blank. With the application of micro seismic monitoring technology is more and more widely, the seismic background noise of the earth as a method for the oil and gas exploration will become a special field of micro seismic monitoring technology of the future. Chinese will continue to in-depth study and understand the application technology of micro earthquakes in the exploration and development of oil and gas.

\section{CONCLUSION}

Micro seismic monitoring technology has obtained widespread attention in China various geological research field. In the energy exploitation, China more focus on resource exploitation efficiency To avoid loss of resources in the mining. Therefore Chinese need to have better technical support in the resource exploitation. China has made some progress in micro seismic monitoring technology, but the core technology is still monopolized by foreign. Micro seismic monitoring technology, as a new geophysical technology in the current China, help solve many problems of mineral in the current security through real-time monitoring of underground rock state of activity and can locate the seismic source. China will devote more energy to study on micro seismic monitoring technology. Therefore Chinese micro seismic monitoring technology will reach the leading level in the future.

\section{REFERENCES}

[1] Chen Huandong, Jiang Fuxing, Zhang Xingmin. Study on nonhomogeneous material's AE by image processing method[J]. Rock and Soil Mechanics, 2004, 60-64.

[2] Chen Shangben, Ben Boyi. Research on Complete Techniques for Prediction and Control of Rock[J]. Bursts Journal of Shandong University of Science and Technology, 2010, 63-66. 
[3] Liu Jianzhong, Feng Tao, Yuan Zengrong, Yang Tao. Observation Of Flood Front And Stress State Around Well[J]. Chinese Journal of Rock Mechanics and Engineering, 2004, 2409-2412.

[4] Zhou jie, Gao Jingwen, He Ping, Han Zhenjun. Application of Microseism and Microfrac Monitoring Tech in Shanshan Oilfield[J]. oil and gas well testing, 2005,62-64.

[5] Wang Yezhong, Deng Jingen, Zhao Zhenfeng, Mu Lijun, Liu Jianan, Tian hong. Downhole Nicroseism Ic Fracture Monitoring Design And Fracturing Results Analysis[J]. Petroleum Geology \& Oilfield Development in Daqing, 2006, 76-78.

[6] Segall P. Earthquakes triggered by fluid extraction[M]. Geology American Elasevier science, 1989, 942-946.

[7] Cui Rongwang. Microseism ic fracture monitoring should be carried out in wells[J]. Petroleum Geology and exploitation in Daqing, 2007, 138-142.

[8] Hu Jinke, Li Gao, Chen Wenke, Yao Yuan, Jiang Yanna. Review on Overseas Exploration and Exploitation of Shale Gas[J]. Journal of Chongqing University of Science and Technology, 2011, 72-75.

[9] Liu Jianan, Ma Hongxing, Mu Lijun, Qiu Xiaolin. The Applicaton of Down Hole Micro-Seismic Fracture Testing Technique in Changqing Oilfield[J]. oil and gas well testing, 2005, 54-56.

[10] liang Bing, zhu Guangsheng. Microseismic monitoring method in Oil and gas exploration and development[M]. Beijing: Petroleum Industry Press, 2004,11.

[11] Lu Hao. Research on the Seismic Phase Identification and the Source Location Based on Oilfield Fracture Microseismic Monitoring[D]. tutor: Teng Jiwen; Lin Jun. Jilin University, 2012,6-7.

[12] Zhang Xingmin, Yu Kemin, Xi Jingde. The research and application of microseismic technology in mine fractured and caving zones monitoring[J]. JOURNAL OF CHINA COAL SOCIETY, 2000, 335-339.

[13] Jing Fuxing, Yang Shuhua, Cheng Yunhai, A study on microseismic monitoring of rock burstin coal mine[J]. CHINESE JOURNAL OF GEOPHYSICS, 2000,1511-1516.

[14] Liang Sheng, Han Liguo, Li Anbang. Application of Microseism Surveillance Technology in Qinjiatun Area[J]. Journal of Jilin University, 2007, 95-98.

[15] Chen Yunhai, Jiang Fuxin, Zhang Xinmin, Mao Zhongyu, Ji Zhenwen. C-Shaped Strata Spatial Structure And Stress Field In Longwall Face Monitored By Micro seismic Monitoring[J]. Chinese Journal of Rock Mechanics and Engineering, 2007, 102-107.

[16] Wang Zhenglei. Detection of weak earthquake signals based on wavelet analysis[J]. Progress in Exploration Geophysics, 2009, 182-185.

[17] Yi Chen, Wu Furong, Li Yalin. The Effect Of The Velocity Calibrated By The Perforation On Microsesmic Location Error[J]. PROGRESS IN GEOPHYSICS, 2013, 1809-1812.

[18] Du Wenjun, Lei Xiange, Huang Jiang. Three Kinds of Arrangements Monitoring Micro-seismic Events Induced by Reservoir Fracturing[J]. Journal of Oil and Gas Technology, 2010, 303-305.

[19] O.H. Barzandji, J.M. Singer, E.D. Rode, W. Leu, J. Plunkett, F. Ghirlanda, K. Akrawi, ADCO. Hydrocarbon Reservoir Detection Using Acoustic/Seismic Spectroscopy Method[J]. Society of Petroleum Engineers, 2002, 34-37. 